\title{
Significance of soluble PD-L1 for malignant tumors
}

\author{
Asanuma K*, Nakamura T, Hagi T, Kita K and Sudo A \\ Department of Orthopedic Surgery, Mie University School of Medicine, Tsu City, Mie, Japan
}

The tumor immune microenvironment has drawn attention as a therapeutic target. One immune suppressive protein, PD-L1 (B7$\mathrm{H} 1$ or CD274), a $40-\mathrm{kDa}$ transmembrane glycoprotein, is known as a primary ligand of PD-1. PD-L1 expression has been observed in active T cells, B cells, NK cells, DCs, monocytes, macrophages, activated vascular endothelial cells, mesenchymal stem cells, and tumor cells [1]. The interaction of PD-L1 and PD-1 can induce T cell tolerance [2], T cell apoptosis [3], and T cell exhaustion [4]. The enhancement of this immune suppressive protein leads to evasion of the host immune response and tumor aggravation. A relationship between high PD-L1 expression in tumor tissues and poor prognosis in various malignant tumors such as non-small cell lung cancer [5], ovarian cancer [6], renal cell carcinoma [7], melanoma [8], breast cancer [9], and soft tissue sarcoma [10] has been reported. Thus, it is recognized that PD-L1 expression affects tumor behavior and prognosis.

The circulating soluble form of PD-L1 (sPD-L1) in blood has attracted much attention. In addition to poor prognosis related to high $\mathrm{PD}-\mathrm{L} 1$ expression in tumors, high sPD-L1 is related to poor prognosis in various cancers such as renal cell carcinoma [11], hepatocellular carcinoma [12], lung cancer [13], gastric cancer [14], and B cell lymphoma [15]. The link between elevated SPD-L1 and poor prognosis indicates that sPD-L1 probably has functional activity. However, its roles have not been fully elucidated. Here, we review the sources and functions of sPD-L1.

Speculated sources of SPD-L1:

1. Cleavage and release from membrane PD-L1

2. Spliced variants

3. Release by cytokines, cell stress, cell injury, or cell death

Chen, et al. reported that sPD-L1 is released into the culture supernatant and could be decreased by a metalloproteinase (MMP) inhibitor [16]. This means that MMP can release the extracellular domain of membrane PD-L1, leading to SPD-L1. The cleavage site of PD-L1 and the function of cleaved PD-L1 by MMP are still unknown and need further study. Zhou, et al. found four splice variants, PD-L1-1, PD-L1-3, PD-L1-9, and PD-L1-12. It is easy to consider that the variants that lack the transmembrane domain (PD-L1-3, PD-L1-9) are released in the culture medium. However, the variant with a transmembrane domain is also secreted and detected in the culture medium (PD-L1-1). These variants are also observed in the plasma of melanoma patients [17]. In addition, cytokines such as interferon gamma, interferon alpha, and TNF-alpha increase the release of these variants into culture medium [17]. At this time, the possible induction of PD-L1 release by cell stress, cell injury, or cell death cannot be excluded.

\section{Functional assessment of sPD-L1}

Functional assessment of sPD-L1 is extremely important. Chen [16]. and Takeuchi [18]. Developed a unique ELISA to detect
sPD-L1. They used a PD-1-Ig fusion protein to capture sPD-L1, which possesses binding capacity to $\mathrm{PD}-1$, instead of a capture antibody. This ELISA detected 29 out of 75 plasma samples from patients with non-small cell lung cancer, and detected sPD-L1 with much higher sensitivity and frequency than conventional ELISA. This sPD-L1 can probably transduce signals into cells by binding membrane PD-1. PD-L1 glycosylation is also important. As deglycosylation of PD-L1 reduces the absorbance of the ELISA, deglycosylated PD-L1 probably cannot bind PD-1. Glycosylation is therefore a critical factor for their interaction [18]. Additionally, spliced variants have also had their functions assessed. The variants reduce the number of activated $\mathrm{CD} 4^{+}$ and $\mathrm{CD} 8^{+} \mathrm{T}$ cells [17]., and one variant induces apoptosis of $\mathrm{CD} 4^{+} \mathrm{T}$ cells more than $\mathrm{CD}^{+}[11]$. The variants possess inhibitory functions against T-cell activation and proliferation [17].

We believe that high PD-L1 in tissues and SPD-L1 are involved in poor prognosis. However, regardless of PD-L1 expression, clinical data indicate that patients receive benefits from checkpoint inhibitor therapy [19-24]. For that reason, we need to develop predictive biomarkers to establish which patients are most likely to benefit from checkpoint blockade. From what we know, released sPD-L1 (at least except for deglycosylated PD-L1) can affect $\mathrm{T}$ cell biological activity, and this means that circulating SPD-L1 has the potential to induce systemic immune suppression. sPD-L1 may be a biomarker for determining the use of checkpoint inhibitors. However, there are many things about sPD-L1 left to be clarified, and these need further study.

\section{References}

1. Zou W, Chen L (2008) Inhibitory B7-family molecules in the tumour microenvironment Nature Reviews Immunology 8: 465-467.

2. Keir ME (2006) Tissue expression of PD-L1 mediates peripheral T cell tolerance. Journal of Experimental Medicine 203: 883-895.

3. Dong H (2002) Tumor-associated B7-H1 promotes T-cell apoptosis: a potential mechanism of immune evasion. Nature medicine 8: 793

4. Blank C, Mackensen A (2007) Contribution of the PD-L1/PD-1 pathway to T-cell exhaustion: an update on implications for chronic infections and tumor evasion. Cancer immunology, immunotherapy 56: 739-745.

5. Wang A (2015) The prognostic value of PD-L1 expression for non-small cell lung cancer patients: a meta-analysis. European journal of surgical oncology 41: 450-456.

6. Darb-Esfahani S (2016) Prognostic impact of programmed cell death-1 (PD-1) and PD-ligand 1 (PD-L1) expression in cancer cells and tumor-infiltrating lymphocytes in ovarian high grade serous carcinoma. Oncotarget 7: 1486.

${ }^{\star}$ Correspondence to: Kunihiro Asanuma, Mie University, Orthopedic Surgery, 2-174, Edobashi, Tsu City, Mie 514-8507, Japan, E-mail: kasanum@gmail.com

Key words: PD-L1, soluble, soft tissue tumor, soft tissue sarcoma, metastasis, prognosis

Received: October 13, 2018; Accepted: October 29, 2018; Published: October 31,2018 
7. Thompson RH, Kuntz SM, Leibovich BC, Dong H, Lohse CM, et al. (2006) Tumor B7-H1 is associated with poor prognosis in renal cell carcinoma patients with long-term follow-up. Cancer Res 66: 3381-3385. [Crossref]

8. Gadiot J (2011) Overall survival and PD-L1 expression in metastasized malignant melanoma. Cancer 117: 2192-2201.

9. Muenst S (2014) Expression of programmed death ligand 1 (PD-L1) is associated with poor prognosis in human breast cancer. Breast cancer research and treatment 146: 1524.

10. Kim C, Kim EK, Jung H, Chon HJ, et al. (2016) Prognostic implications of PD-L1 expression in patients with soft tissue sarcoma. BMC Cancer 16: 434. [Crossref]

11. Frigola X (2011) Identification of a soluble form of B7-H1 that retains immunosuppressive activity and is associated with aggressive renal cell carcinoma. Clinical Cancer Research 17: 1915-1923.

12. Finkelmeier F (2016) High levels of the soluble programmed death-ligand (sPD-L1) identify hepatocellular carcinoma patients with a poor prognosis. Eur J Cancer 59:152159.

13. Okuma Y, Hosomi Y, Nakahara Y, Watanabe K, Sagawa Y, et al. (2017) High plasma levels of soluble programmed cell death ligand 1 are prognostic for reduced survival in advanced lung cancer. Lung Cancer 104: 1-6. [Crossref]

14. Takahashi N, Iwasa S, Sasaki Y, Shoji H, Honma Y, et al. (2016) Serum levels of soluble programmed cell death ligand 1 as a prognostic factor on the first-line treatment of metastatic or recurrent gastric cancer. J Cancer Res Clin Oncol 142: 1727-1738. [Crossref]

15. Rossille D (2014) High level of soluble programmed cell death ligand 1 in blood impacts overall survival in aggressive diffuse large B-Cell lymphoma: results from a French multicenter clinical trial. Leukemia 28: 2367-75.
16. Chen Y (2011) Development of a sandwich ELISA for evaluating soluble PD-L1 (CD274) in human sera of different ages as well as supernatants of PD-L1+ cell lines. Cytokine 56: 231-238.

17. Zhou J (2017) Soluble PD-L1 as a Biomarker in Malignant Melanoma Treated with Checkpoint Blockade. Cancer Immunol Res 5: 480-492.

18. Takeuchi M, Doi T, Obayashi K, Hirai A, Yoneda K, et al. (2018) Soluble PD-L1 with PD-1-binding capacity exists in the plasma of patients with non-small cell lung cancer. Immunol Lett 196: 155-160. [Crossref]

19. Kaufman HL (2016) Avelumab in patients with chemotherapy-refractory metastatic Merkel cell carcinoma: a multicentre, single-group, open-label, phase 2 trial. The Lancet Oncology 17: 1374-1385.

20. Herbst RS (2016) Pembrolizumab versus docetaxel for previously treated, PD-L1positive, advanced non-small-cell lung cancer (KEYNOTE-010): a randomised controlled trial. The Lancet 387: 1540-1550.

21. Weber JS (2015) Nivolumab versus chemotherapy in patients with advanced melanoma who progressed after anti-CTLA-4 treatment (CheckMate 037): a randomised, controlled, open-label, phase 3 trial. The Lancet Oncology 16: 375-384.

22. Rosenberg JE (2016) Atezolizumab in patients with locally advanced and metastatic urothelial carcinoma who have progressed following treatment with platinum-based chemotherapy: a single-arm, multicentre, phase 2 trial. The Lancet 387:1909-1920.

23. D'Angelo SP, Mahoney MR, Van Tine BA, Atkins J, Milhem MM, et al. (2018) Nivolumab with or without ipilimumab treatment for metastatic sarcoma (Alliance A091401): two open-label, non-comparative, randomised, phase 2 trials. Lancet Oncol 19: 416-426. [Crossref]

24. Tawbi HA, Burgess M, Bolejack V, Van Tine BA, Schuetze SM, et al. (2017) Pembrolizumab in advanced soft-tissue sarcoma and bone sarcoma (SARC028): a multicentre, two-cohort, single-arm, open-label, phase 2 trial. Lancet Oncol 18: 14931501. [Crossref]

Copyright: (C2018 Asanuma K. This is an open-access article distributed under the terms of the Creative Commons Attribution License, which permits unrestricted use, distribution, and reproduction in any medium, provided the original author and source are credited. 